\title{
Isolation of Microsatellite Loci in Sceloporus grammicus (Squamata, Phrynosomatidae)
}

\author{
Patrick H. Degnan* and Elisabeth Arévalo ${ }^{\dagger}$ \\ Department of Biology \\ Providence College \\ Providence, RI 02918-0001 USA
}

Received: November 18, 2003

Accepted: December 18, 2003

\begin{abstract}
The mesquite lizard (Sceloporus grammicus) exhibits multiple Robertsonian chromosomal rearrangements (mainly centric fissions) resulting in several cytotypes. In a transitional environment from oak-pine forests to a drier xeric habitat in central Mexico, two cytotypes (F5: $2 \mathrm{n}$ $=34$ and FM2: $2 n=46$ ) are known to hybridize. A partial genomic library was constructed from $S$. grammicus genomic DNA and then screened for microsatellites. Microsatellites are short tandem nucleotide repeats that have near universal occurrence in all eukaryotic genomes. Microsatellites exhibit variable length polymorphisms that can be characterized and utilized as genetic markers for population studies. Thirteen microsatellite arrays were isolated from the $S$. grammicus genomic library and PCR primers were designed in the flanking regions for the amplification of these alleles. These microsatellite loci would be the primary tool used to answer behavioral, ecological, chromosomal and evolutionary questions that influence the maintenance of this hybrid zone.
\end{abstract}

\section{INTRODUCTION}

The phrynosomatid lizards of the Sceloporus grammicus complex represent a ubiquitous species group ranging throughout Mexico and into southern Texas, present exclusively at high elevations, from \pm 2400 $3200 \mathrm{~m}$ (or $\pm 7000-1000 \mathrm{ft}$ ) [1, 2]. Lizards of the genus Sceloporus are one of the most species-rich reptiles in North America with nearly 80 morphologically and ecologically distinct species, many of which occur sympatrically $[3,4]$. The $S$. grammicus species complex is characterized by

* Current address: Josephine Bay Paul Center for Comparative Molecular Biology and Evolution, Marine Biological Laboratory, 7 MBL Street, Woods Hole, MA 02543 USA.

$\dagger$ Corresponding author:

Phone: 401/865-2158

Fax:401/865-1438

E-mail: earevalo@providence.edu ovoviviparous females producing a single brood per year and evident sexual dimorphism including, differences in the coloration of ventral scales and overall larger body size in males. As is the case with most lizard species, males are polygynous, dominate territories that include two to six females and occasionally satellite or subordinate males [5]. Their abundance results in ease of observation and collection. Thus, they have proven to be an excellent model for the research of phylogenetic, ecological, chromosomal, evolutionary and population structure.

Members of the $S$. grammicus complex are currently classified as incipient species, and have been characterized by eight distinct chromosomal races or cytotypes [6,7]. The variation between the chromosomal races is mainly due to Robertsonian macrochromosmal fissions [3, 8] resulting in individuals with karyotypes that range from $2 n=32$ to $2 n=46$ [6,9-11]. 


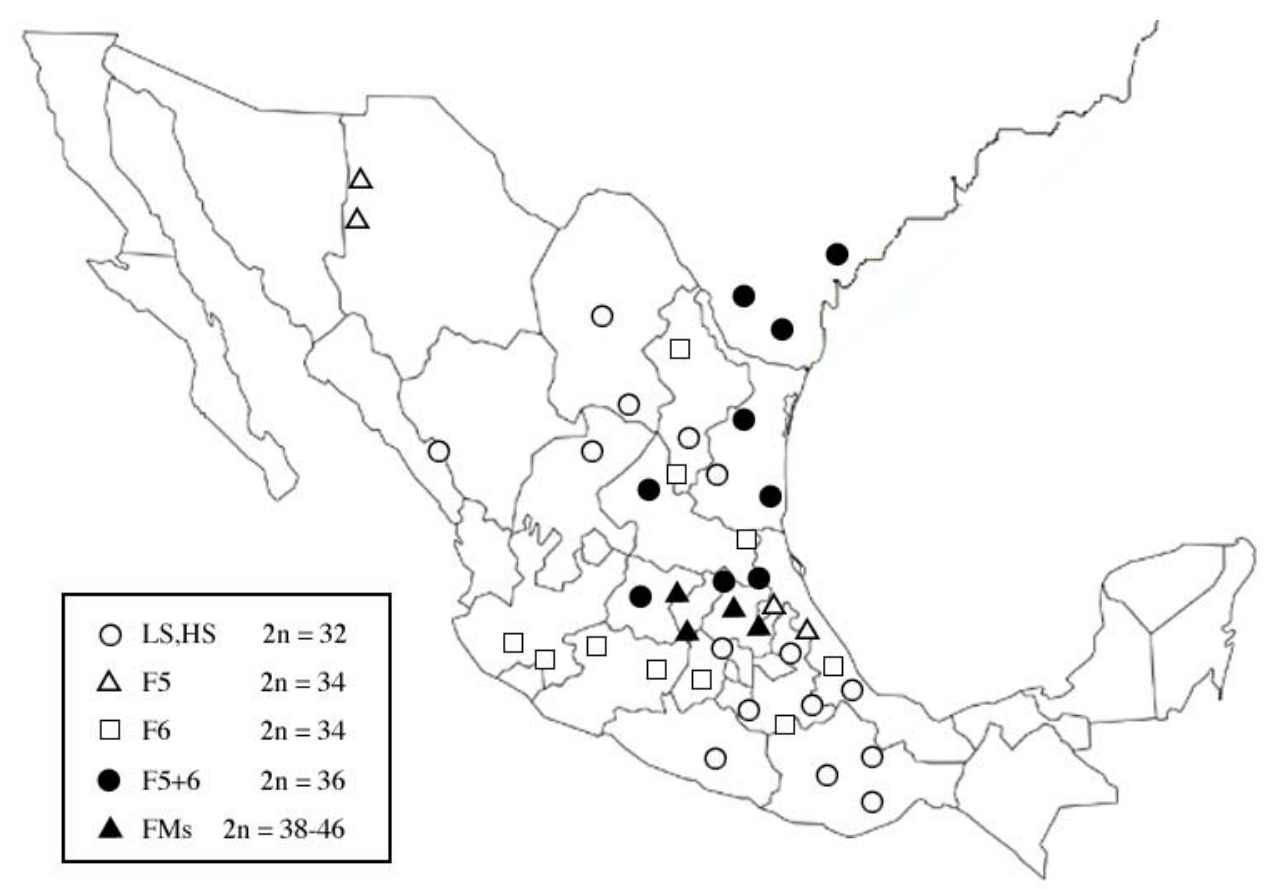

Figure 1. Map of Mexico indicating the widely distributed populations of Sceloporus grammicus cytotypes that have been sampled.

The standard type (S), $2 n=32$ cytotype has been supported as being the ancestral or primitive group and the centric fissions of few to all chromosomes resulted in the more derived cytotypes LS, HS, F5, F6, F5+F6, FM1, FM2 and FM3 (Figure 1) [8, 10]. The ancestral cytotype is composed of six pairs of macrochromosomes and ten pairs of microchomosomes in females and $9+Y$ in males. As an example of the cytotype variability the F6 population differs from the $S$ type by possessing a fixed fission of chromosome pair 6 resulting in a $2 n=34[7$, 8].

Regions where there are relatively similar populations of individuals, that possess distinct genetic differences (i.e. cytotypes) and who have shared territories resulting in the capability for mating, can result in hybridization [11,12]. The high degree of chromosomal polytypy within and between populations of $S$. grammicus is rare among non-mammalian vertebrates, and creates a unique and accessible model for examining vertebrate hybrid zones [10, 11]. Individuals of $\mathrm{S}$. grammicus that have different cytotypes can still mate and produce viable and fertile $F_{1}$ generations [2, 13]. At least seven distinct narrow hybrid zones of $S$. grammicus cytotypes have been described in Central Mexico [10, 13]. Further studies at one of the described hybrid zones $[13,14]$ showed normal disjunction and balanced chromosomal segregation during meiosis of $\mathrm{F} 5$ heterozygotes. The hybrid zone near Tulancingo, Hidalgo in central Mexico (Figure 2) encompasses a hybrid zone that involves populations of the F5 cytotype $(2 n=34)$ and the most derived FM2 $(2 n=46)$ cytotype $[2,3,11]$.

Although the two parental cytotypes have shown a statistically significant preference for specific habitats (F5: oak forest and FM2: more xeric environments), they continue to exhibit free association and hybridization within a transitional environment (Figure 2) [2, 11]. The cytonuclear structure of the F5 cytotype is characterized by fixation of the bi-armed chromosome pairs 1,2 and 6 , as well as fixation of a centric fission of chromosome 5 . FM2 races near the town of Tulancingo possess multiple fissions, resulting in 
acrocentric fixation of chromosome pairs 1, 2, 3, 5 and 6 [11]. When these two parental cytotypes cross and form an $F_{1}$ hybrid it exhibits a significant reduction in fitness [13]. There is a loss of fecundity and a reduction in clutch size in $\mathrm{F}_{1}$ females, and meiotic nondisjunction, and higher levels of aneuploid sperm in $F_{1}$ males $[11,13,15]$. These studies $[11,16]$ indicate that mating occurs with approximate randomness within local neighborhoods. However, it has been shown that selective backcrosses of the F1 generations to parental genotypes can help stabilize chromosomal hybrids $[11,13,15]$. A clear reproductive cost is incurred in the production of hybrids, in comparison to pure and backcrossed individuals, our group interest is to assess the underlying means of sexual selection and mating success within these hybrid zones. Powerful molecular markers are necessary to help characterize these complex male and female interactions.

Microsatellites have proven to be ideal means to enable the development of detailed population models based on frequency and heterozygosity of identified alleles $[17,18]$. In a population where basic phenotypes and morphological characters become indistinct and prove inconclusive when analyzing various evolutionary and behavioral trends, microsatellites can serve as highly polymorphic genetic markers. Microsatellites, also known as simple sequence repeats (SSR) or variable number of tandem repeats (VNTR), are regions of DNA that exhibit short repetitive sequence motifs $[17,19,20]$. These motifs are most often present in 2-6 nucleotide sequences (i.e., CA, AAT, CGGG) that are repeated for anywhere from 5-50 times [17, 21].

The highly repetitious nature of these regions result in an increased rate of mutations due to slip-strand mispairing, decreases in the efficiency of mismatch repair (MMR) systems and less frequently, unequal crossing over during replication phases [22-24]. Microsatellites are not reserved to non-coding '5' and 3' untranslated regions and introns, but are also found in eukaryotic exons [17, 25]. Research has also shown differential stability of microsatellites across interspecific and intraspecific relationships as well as between the sexes [25]. Somatic microsatellite mutations have also been strongly implicated in several human diseases suggesting failure of the MMR systems and or replication mechanisms [25, 26].

It has been estimated that during DNA replication in humans that point mutations occur at a rate of $10^{-9}-10^{-10}$ events per locus [19]. Thus, evolution and accumulation of distinct mutations in the DNA sequence by this means of mutation is very slow. Mutations within microsatellite arrays though, occur at a much higher rate $10^{-3}$ in humans and $10^{-2}$ in E. coli [19].
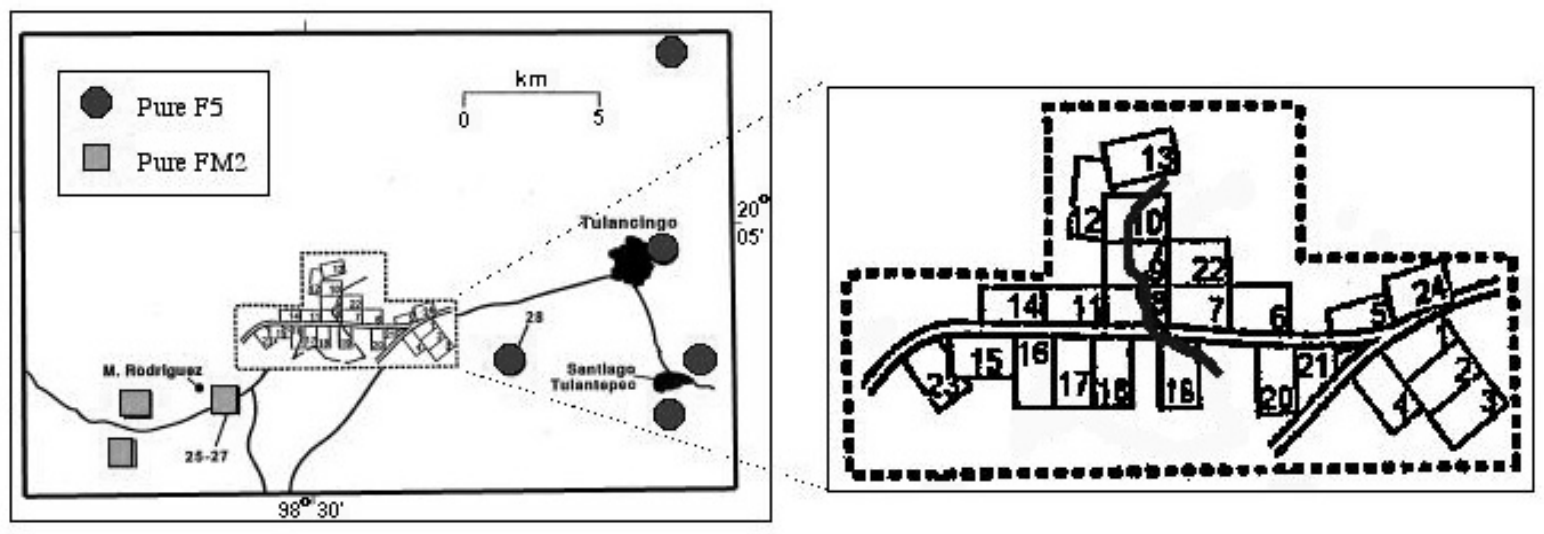

Figure 2. Map of central Mexico near Tulancingo, Hidalgo. The black filled circles indicate pure F5 cytotype sample populations and gray filled squares indicate pure FM2 cytotype sample populations in the surrounding regions. Inset detail shows the 28 quadrates across the Tulancingo Hybrid Zone. The dark gray line indicates the central region of contact between the two chromosomal races. 
The increased propensity for mutations to occur at microsatellite loci can create anywhere from 4-20 polymorphic trinucleotide alleles for a single locus [21]. Microsatellites are randomly distributed within approximately $5 \%$ of eukaryotic genomes [17-19, 27]. The near universal occurrence combined with interspecific homology creates a statistical correlation between microsatellites and evolutionary relationships. In studies pertaining to species groups that exhibit little mtDNA or allozyme heterozygosity, microsatellites become the ideal Mendelian, codominant, selectively neutral and highly polymorphic genetic marker $[21,28]$. This project is designed to develop a library of microsatellites for Sceloporus grammicus to help further the understanding of population structure, parental and behavioral studies in the Tulancingo hybrid zone.

\section{METHODS AND MATERIALS}

\section{a. Genomic DNA Extraction and Preparation}

For the development of the Sceloporus grammicus microsatellite library a modified version of the protocol outlined by Strassmann et al. [21] was followed. The genomic DNA utilized to screen for microsatellites was obtained from two adult male lizards independently collected from different localities in central Mexico. The first male was an ' $S$ ' $(2 n=32)$ and the second male was an ' $F 5$ ' $(2 n=34)$ collected near Tulancingo, Hidalgo. According to

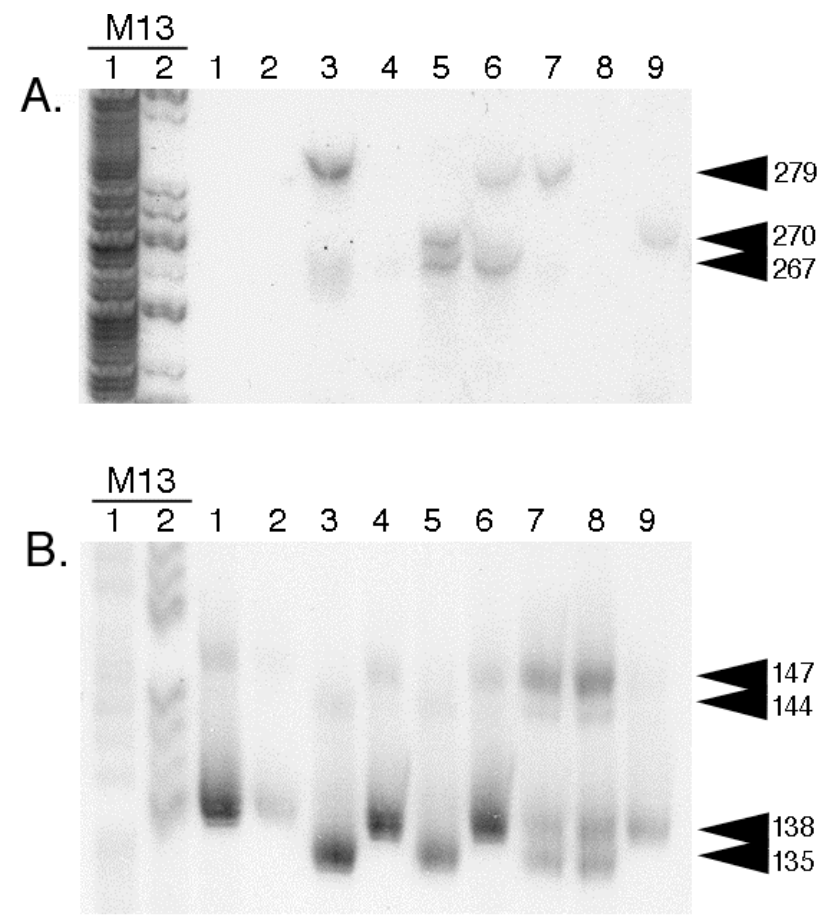

Figure 3. Autoradiographs of PCR amplifications of two microsatellite loci for nine specimens from the Tulancingo hybrid zone A.) Sgram309AGG with allele sizes: 267, 270, 279 and B.) Sgram312GAC with allele sizes: $135,138,144,147$. The DNA samples were isolated from specimens with collection numbers: 1-JWS2103, 2-JWS2092, 3-JWS2094, 4-JWS2095, 5JWS2104, 6-JWS2015, 7-JWS2016, 8-JWS2017, 9-JWS2018. These lizards were collected by J.W. Sites in 1991 and voucher specimens are available for inspection at UNAM-MZFC. Bacteriophage M13mp18 control DNA ladder was used to size alleles. Sequencing reactions with labeled bases $A, G$ and $T$ were mixed together into on single lane (M13-1) and $C$ was run in a separate lane (M13-2) creating a unique banding pattern for easy microsatellite size determination. 


\begin{tabular}{ll}
\hline Oligonucleotide & Annealing T $\left({ }^{\circ} \mathrm{C}\right)$ \\
\hline AAT & 50 \\
AAC,AAG,TAG,CAT & 55 \\
CAG,CAC,GAC,GAG & 65 \\
CCG & 70 \\
\hline
\end{tabular}

Table 1. The series of oligonucleotide primers and their specific trinucleotide repeat motifs and annealing temperature utilized to screen colonies bound to hybridization membranes.

Strassmann's protocol, $100 \mathrm{mg}$ of the frozen muscle tissue was ground and incubated in a solution of STE buffer, $10 \mathrm{mg} / \mathrm{ml}$ of Proteinase $\mathrm{K}$, and $20 \%$ SDS, followed with phenol and chloroform extractions. DNA was digested with Sau3A (New England Biolabs) and fragments ranging from 200-1000bp were size-selected by excision from an agarose gel.

\section{b. Plasmid Ligation and Colony Screening}

The fragments were cloned into a pZero $^{\mathrm{TM}}-2$ vector with the use of Zero background cloning kit. (Invitrogen, Inc.) Colonies were counted and transferred to nylon hybridization transfer membranes (PE Life Sciences). The adherent cells were denatured, neutralized and baked at $65^{\circ} \mathrm{C}$ for three hours. Ten oligonucleotides $\left(\gamma^{33} \mathrm{P}\right.$ radioactively labeled) were used as probes for the hybridization. The positive colonies were visualized with the use of autoradiography. Plasmid preps were completed with the QIAprep Spin Mini Plasmid Prep kit (QIAGEN). Frozen permanents of clones were made in equal volumes glycerol and SOC media and stored at $-80^{\circ} \mathrm{C}$.

c. Microsatellite Sequencing

All positive clones were sequenced on the ABI Prism 377 DNA sequencer with BigDye fluorescently labeled dye terminators (Applied Biosystems). DNA for sequence reactions contained $0.5 \mu \mathrm{g}$ of plasmid dsDNA and $3.2 \mathrm{pmol}$ of the T7 primer. Sequences were then analyzed and PCR primers were designed using MacVector v6.5 (Oxford Molecular).

\section{RESULTS}

The modified Strassmann et al. [21] protocol was performed in three replications resulting in the creation of three partial genomic libraries. Hybridization of the membranes led to the identification of 45 positive clones from a total of 4,430 colonies (Table 2). Sequencing of the positives confirmed that 15 contained microsatellites, 13 of which had unique flanking sequences, allowing for primer design (Table 3). Of these sequences only three contained perfect repeat motifs. Of the remaining sequences one contained a single base deletion, four others contained single base substitutions, and the remaining clones had multiple imperfections of the microsatellite region (Table 3). The microsatellite arrays range from 5-21 repeat units and have a mean of 13 repeat units. Preliminary data from PCR amplifications, suggests that

\begin{tabular}{|c|c|c|c|c|c|}
\hline & \multicolumn{3}{|c|}{ Number of } & \multirow{2}{*}{$\begin{array}{c}\% \text { of } \\
\text { Confirmed } \\
\text { Positives } \\
\end{array}$} & \multirow{2}{*}{$\begin{array}{c}\% \text { of } \\
\text { Colonies } \\
\text { Positive }\end{array}$} \\
\hline & Colonies & Positives & Confirmed & & \\
\hline Library 1 & 698 & 7 & 5 & $71 \%$ & $0.72 \%$ \\
\hline Library 2 & 2394 & 21 & 2 & $10 \%$ & $0.08 \%$ \\
\hline Library 3 & 1338 & 17 & 8 & $47 \%$ & $0.60 \%$ \\
\hline
\end{tabular}

Table 2. Comparative efficiencies of clones generated in the three partial genomic libraries screened for microsatellites for the lizard species S. grammicus. 


\begin{tabular}{|c|c|c|c|c|}
\hline Locus & Clone & Simple Sequence & Motif & $\begin{array}{c}\text { GenBank Accession } \\
\text { Number }\end{array}$ \\
\hline$\overline{\text { Sgram101A }}$ & $1-01-5514$ & $(\mathrm{AGG})_{2}, \mathrm{AAG}, \mathrm{GGC}, \mathrm{ACC},(\mathrm{AGG})_{3}$ & AGG & AY386346 \\
\hline Sgram102A & $1-02-5514$ & $\mathrm{AAC}, \mathrm{AAG},(\mathrm{AAC})_{3}$ & AAC & AY386347 \\
\hline Sgram104A & $1-04-5016$ & $(\mathrm{AAT})_{14}, \mathrm{AA}, \mathrm{AAT}$ & ATT & AY386348 \\
\hline Sgram106A & $1-06-5018$ & $(\mathrm{AAT})_{3}, \mathrm{TAT},(\mathrm{AAT})_{10}$ & AAT & AY386349 \\
\hline Sgram107A & $1-07-5518$ & $\mathrm{GTT}, \mathrm{GTC},(\mathrm{GTT})_{4}$ & AAC & AY386350 \\
\hline Sgram202C & $2-02-65 Z$ & $(\mathrm{CAG})_{3}, \mathrm{G}, \mathrm{CAG}, \mathrm{A},(\mathrm{CAG})_{2}$ & CAG & AY386351 \\
\hline Sgram205A & $2-05-65 Z$ & AGG,AAG,AGG,ATG,(AGG) & AGG & AY386352 \\
\hline Sgram309A & $3-09-655$ & GAG, GAA, GGA,(GAG) 6 & AGG & AY386353 \\
\hline Sgram310C & $3-10-656$ & $(\mathrm{CAC})_{9}$ & CAC & AY386354 \\
\hline Sgram312G & $3-12-656$ & CAG,CTG, $(\mathrm{CAG})_{9}$ & GAC & AY386355 \\
\hline Sgram313A & $3-13-508$ & $(\mathrm{AAT})_{14}$ & AAT & AY386356 \\
\hline Sgram314A & $3-14-508$ & $(\mathrm{AAT})_{6}, \mathrm{AGT}(\mathrm{AAT})_{4}, \mathrm{AT}(\mathrm{AAT})_{9}$ & AAT & AY386357 \\
\hline Sgram316A & $3-16-509$ & $(\mathrm{AAT})_{11}$ & AAT & AY386358 \\
\hline
\end{tabular}

Table 3. Confirmed microsatellite containing clones isolated from S. grammicus partial genomic library. Specific microsatellite arrays, motifs and GenBank accession numbers listed. Clones identified by library, clone number, hybridization temperature and gel number (1-XX-YYZ).

these loci are polymorphic across a narrow sampling of individuals (Figure 3 ). Primer sequences and PCR conditions are available upon request (from EA).

\section{DISCUSSION}

At the end of this study we were able to isolate 15 microsatellites from Sceloporus grammicus with $\geq 5$ repeat units. The relative success in isolating microsatellites from each of the three replications varied widely both in respect to the number of positives collected from the total colonies screened and percentage of colonies confirmed (Table 2). Our results were compared against similar studies across both invertebrates and vertebrates (Table 4); 


\begin{tabular}{|c|c|c|c|c|c|c|c|c|c|}
\hline & Total & Insert & Probed & Positives & Sequenced & Confirmed & Primers & $\%$ of & $\%$ of \\
\hline & Number of & Size (kb) & Oligos & & & & & Colonies & Seq. \\
\hline & clones & & & & & & & Positive & Confirm \\
\hline \multirow{2}{*}{$\begin{array}{l}\text { Invertebrates: } \\
\text { Meganyctiphanes } \\
\text { norvegica }^{1}\end{array}$} & & & & & & & & & \\
\hline & 5,000 & $0.2-0.8$ & $(17)^{\mathrm{a}}$ & 12 & 12 & 12 & 6 & $0.24 \%$ & $100 \%$ \\
\hline Penaeus monodon ${ }^{2}$ & 2,417 & $0.3-1.0$ & $(9)^{\mathrm{b}}$ & 406 & 253 & 253 & 129 & $16.80 \%$ & $100 \%$ \\
\hline Polistes annularis ${ }^{3}$ & $5,000-10,000$ & $0.4-0.6$ & $(10)^{c}$ & 120 & 39 & 33 & 6 & $1.2-2.4 \%$ & $85 \%$ \\
\hline $\begin{array}{l}\text { Pseudomyrmex } \\
\text { pallidus }^{4}\end{array}$ & 15,000 & $0.3-0.6$ & $(6)^{d}$ & 279 & 102 & 53 & 9 & $1.86 \%$ & $52 \%$ \\
\hline Formica lugubris $^{5}$ & 2,400 & $0.3-0.5$ & $\mathrm{CA} / \mathrm{GT}$ & 21 & 17 & 17 & 8 & $0.88 \%$ & $100 \%$ \\
\hline \multicolumn{10}{|l|}{ Vertebrates: } \\
\hline Molothrus ater ${ }^{6}$ & 1,536 & $19.5-53.2$ & $(17)^{\mathrm{e}}$ & 535 & - & 535 & - & $34.83 \%$ & - \\
\hline $\begin{array}{l}\text { Ctenophorus } \\
\text { ornatus }^{7}\end{array}$ & 1,300 & $0.2-0.7$ & $(6)^{f}$ & 14 & 12 & 10 & 7 & $1.08 \%$ & $83 \%$ \\
\hline $\begin{array}{l}\text { Sceloporus } \\
\text { grammicus }\end{array}$ & 4,430 & $0.2-0.5$ & (10) & 45 & 45 & 15 & 13 & $1.02 \%$ & $33 \%$ \\
\hline \multicolumn{10}{|l|}{ Chelonia mydas $^{8}$} \\
\hline $\begin{array}{l}\text { Caretta caretta }^{8} \\
\text { Eretmochelys } \\
\text { imbricata }^{8}\end{array}$ & 64,000 & $0.35-0.5$ & $\mathrm{CA} / \mathrm{GT}$ & 142 & 63 & 24 & 7 & $0.22 \%$ & $38 \%$ \\
\hline Porcine $^{9}$ & 10,000 & $0.2-0.4$ & $\mathrm{CA} / \mathrm{GT}$ & 87 & 26 & 26 & 6 & $0.87 \%$ & $100 \%$ \\
\hline Homo sapiens ${ }^{10}$ & 12,014 & $0.3-0.5$ & $\mathrm{CA} / \mathrm{GT}$ & 2,995 & 12,014 & 2,506 & 2,506 & $24.9 \%$ & $21 \%$ \\
\hline
\end{tabular}

Table 4. A comparison of the efficiency of microsatellite isolation from our S. grammicus partial genomic libraries to that of related studies from both invertebrates and vertebrates. Oligonucleotides used in studies screening for more than 2 motifs: ${ }^{a} G G A T, A G C T, A G A C, T A C T$, TAGT, ATCT, AAAG, AAAC, AAAT, TGT, TCT, TAG, TAT, TAG, TAT, AC, AG, ' $A G$, TG, GAA, GAC, CAT, TAC, GACA, GATA, TCAG, 'AAT, AAC, AAG, TAG, CAT, CAG, CAC, GAC, GAG, CCG, 'AAT, AAC, AAG, CAT, TAG, GAG, 'GATA, AGC, GTT, AGT, CGT, CCT, AAT, ACC, AAG, GAT, CCG, AT, CG, GT, CT, C, A, ${ }^{\dagger} A A G$, AAT, AAC, AT, CA, AG. Studies cited: ${ }^{1}$ [42], ${ }^{2}[29],{ }^{3}[28],{ }^{4}[43],{ }^{5}[44],{ }^{6}[31],{ }^{7}[45],{ }^{8}[46],{ }^{9}[47],{ }^{10}[48]$.

the low yield in our study is consistent with the majority of other studies reviewed. Seven of the other ten literature sources listed in Table 4 collected $<2.5 \%$ of positives from the total number of colonies screened and six of the sources had $<100 \%$ sequence confirmation. The variability seen in microsatellite isolation is correlated to the relative efficiency of protocols used by particular laboratories, the number and type of oligos used, as well as the overall density of microsatellites in particular genomes.

The increased interest in microsatellites for genetic studies has led to the development of several different strategies to provide the most efficient means of isolating tandem repeat arrays. The choices laboratories make are dependent upon those which are cost effective and those that are available. DNA enrichment protocols can lead to a 10-15-fold increase in fragments containing microsatellite arrays prior to insertion into plasmid vectors [29, 30]. Also high-density gridded arrays of cosmid clones allow for the insertion of larger genomic fragments (19.1-53.2kb) into the vector. This allows for a greater quantity of genomic DNA to be processed in a fewer number of clones [31]. Other research groups have developed non-radioactive labeling methods, such as Boehringer Mannheim's digoxigenin labeled oligonucleotides that require chemiluminesence or colorimetric detection. Another simple and useful 
strategy is to run a southern blot to double check clones prior to sequencing [21, 28].

The net yield of microsatellites isolated from any subject not only varies due to differences in experimental protocol, but also human error. Often errors in stringency of the hybridization solutions and selection of colonies based on autoradiographs will lead to false positives ([32]: and personal correspondence J. J. Agresti Medical Research Council: Molecular Oncology Group, and A. Ball South Carolina Department of National Resources). During the third genomic library screening with the $55^{\circ} \mathrm{C}$ oligos there were a high number of false positives generated, that when sequenced were shown to clearly lack microsatellites. These results could very well be directly correlated to the stringency of the hybridization solutions.

Inherent difficulty in microsatellite library construction also arises due to variable densities and frequencies of specific microsatellite motifs. Reptilian genomes have a general size range of $0.9 \times 10^{9}$ $4.7 \times 10^{9}$ base pairs $(2.10-10.44 \mathrm{pg})$ [33]. The partial genomic library developed in this study only analyzed an estimated $1550 \mathrm{~kb} \pm$ $919 \mathrm{~kb}$ of $S$. grammicus DNA. Results obtained by Olmo [34] showed that the closely related species of $S$. grammicus, $S$. occidentalis (Western fence lizard) has a genome consisting of $2.02 \times 10^{9} \mathrm{bp}$ of DNA (4.72pg). Sceloporus occidentalis is a member of the $S$. horridus species group and like $S$. grammicus is a member of the monophyletic "large bodied and large scaled" (LB/LS) radiation within the genus Sceloporous [35, 36]. Based on Olmo's genome estimate, only $0.08 \%$ of the $\sim 2.02 \times 10^{9} \mathrm{bp}(4.72 \mathrm{pg})$ was screened for microsatellites. Research in a related Sceloporine LB/LS lizard S. jarrovii, was able to isolate fifteen microsatellites with $(C A)_{n},(C T)_{n}$ and $(A A T)_{n}$ motifs [4]. Nine of these loci proved to be polymorphic and eight of the nine were dinucleotide repeats. Their library construction protocol utilized an enrichment step and the quantity of DNA screened is not indicated, so a comparable estimate of average Sceloporine microsatellite density is not possible.

If our estimate of genomic DNA screened is fairly accurate, it would indicate that S. grammicus has only $\sim 20,000$ trinucleotide microsatellite loci or one every
$103 \mathrm{~kb}$. This is a striking disparity when compared to the human genome, which is comprised of $3.0 \times 10^{9} \mathrm{bp}(7.00 \mathrm{pg})$ and has been estimated to contain $\sim 500,000$ loci or one microsatellite every $6 \mathrm{~kb}$ [37]. The average avian genome has been shown not only to be smaller but to also have lower densities of microsatellites [38]. Primmer et al. [38] found that birds have an average density of one microsatellite every $39 \mathrm{~kb}$ or $\sim 30,000-70,000$ loci, based on a genome size estimate of $1.2 \times 10^{9} \mathrm{bp}(2.50 \mathrm{pg})$. In the brown headed cowbird, Molothrus ater, Longmire et al. [31] found that it's microsatellite density was only one repeat motif every $89 \mathrm{~kb}$. Invertebrates have been characterized as having relatively low microsatellite abundance as well [20], but interestingly in at least 2 species of shrimp, particularly long microsatellites have been recovered ( $\leq 85$ repeat units: $[29,39]$ ).

In general mammals, plants and birds have also been discovered to have certain characteristic microsatellite motifs that occur more frequently in their genomes. Mammals (rats and humans) exhibit a prevalence for $(C A)_{n},(A)_{n},(A A A T)_{n}$, and $(A G)_{n}$ repeat motifs, while in plants $(C A)_{n}$ is rare but $(A A)_{n}$ and $(\mathrm{AT})_{\mathrm{n}}$ are very common, and birds have a higher frequency of mono- and tetranucleotide repeats than di- and trinucleotide repeats $[32,37,38]$. The relative low yield of positive colonies from research presented in Table 4, could correspond in certain cases to the utilization of only a single set of oligos, which limits the potential range of microsatellites that are detected. Apart from variation in microsatellite frequency and repeats, certain plant and animal species have also been shown to have difficult to obtain microsatellites $[23,40]$.

Despite the low density of microsatellites derived from some of the libraries (Table 4), the primers that were developed all proved to amplify polymorphic loci that were instrumental in analyzing various intra- and interspecific populations. The primers that we have developed should provide sufficient polymorphic markers for further population studies of $S$. grammicus from the Tulancingo hybrid zone, as well as from other areas of interest. Twelve of our primers have successfully amplified loci of expected size ranges across several lineages (Figure 3) and we are in the 
process of assessing the levels of polymorphisms for the different loci. Zamudio and Wieczorek [4] screened their $S$. jarrovii primers across several related taxons, but state that the primers will most likely only show significant polymorphisms across the $S$. torquatus group.

There are many evolutionary, developmental, biochemical, and behavioral questions that are still unanswered concerning the $S$. grammicus complex. Microsatellites have the potential of expressing diagnostic polymorphisms, that used in conjunction with identified chromosomal and isozyme polymorphisms with fixed genetic differences, we will develop the necessary power to distinguish the eight chromosomal races. They also have the power to assess the effect genotypes have on mate selection, especially those that result in successful backcrosses that stabilize cytonuclear structures and restore female fecundity. These loci will also be used to estimate the reproductive cost for hybrid males, by evaluating their ability to act as a dominant, territorial male and control a harem of females. Allelic frequencies will be estimated with the use of Goodnight and Queller's Relatedness software (v5.0.4) [41]. These frequencies will reveal if females are singly or multiply mated and provide estimates of male mating success within territories. After the completion of both parental and offspring genotypes, correlations between cytotypes and male territory distributions will be able to establish a size or quality difference between hybrid and pure males. These microsatellites will provide a new means of characterizing this species complex that will supplement data already collected using morphological, mtDNA and allozyme markers.

\section{ACKNOWLEDGEMENTS}

The authors would like to thank Thomas Earl, Amanda Townsend, Mike MacPhee, Ruth Lambert, George Dekki, and Brendan Lefebvre for assistance in the lab. We greatly appreciate the contribution of lizard specimens by Jack W. Sites Jr. We would also like to thank anonymous reviewers for their insightful comments on the manuscript. This research was possible through the support of the NSF:IBN9900975.

\section{REFERENCES}

1. Sites, J.W., Jr. and J.R. Dixon, $A$ new subspecies of the iguanid lizard, Sceloporus grammicus from northeastern Mexico, with comments on its evolutionary implications and the status of the S. g. disparilis. J Hepatol, 1981. 15: p. 59-69.

2. Arévalo, E., et al., Population cytogenetics and evolution of the Sceloporus grammicus complex (Iguanidae) in central Mexico. Herpetological Monographs, 1991. 5: p. 79-115.

3. Hall, W.P., Modes of speciation and evolution in the Sceloporine Iguanid lizards., in Advances in Herpetology and Evolutionary Biology. Essays in Honor of Ernest E. Williams, A.G.J. Rhodin and K. Miyata, Editors. 1983. Museum of Comparative Zoology: Cambridge, MA. p. 643-679.

4. Zamudio, K.R. and A.M. Wieczorek, Microsatellites for studies of ecology, behaviour, and evolution in Yarrow's spiny lizard (Sceloporus jarrovii). Mol Ecol, 2000. 9(10): p. 1667-1669.

5. Stamps, J.A., Sexual selection, sexual dimorphism and territoriality, in Lizard Ecology: Studies of a Model Organism, R.B. Huey, E.R. Pianka, and T.W. Schoener, Editors. 1983, Harvard University Press: Cambridge, MA. p. 169-204.

6. Sites, J.W., Jr., P. Thompson, and C.A. Porter, Cascading Chromosomal Speciation in Lizards: A Second Look. Pacific Science, 1988. 42: p. 89-104.

7. Arévalo, E., S.K. Davis, and J.W.S. JR., Mitochondrial DNA sequence divergence and phylogenetic relationships among eight chromosome races of the Sceloporus grammicus complex (Phrynosomatidae) in central Mexico. Syst. Biol., 1994. 43: p. 387418.

8. Hall, W.P. and R.K. Selander, Hybridization of karyotypically differentiated populations in the Sceloporus grammicus complex (Iguanidae). Evolution, 1973. 27: p. 226242. 
9. Porter, C.A. and J.W. Sites, Jr., Normal disjunction in Robertsonian heterozygotes from a highly polymorphic lizard population. Cytogenet Cell Genet, 1985. 39: p. 250-257.

10. Porter, C.A. and J.W. Sites, Jr., Evolution of Sceloporus grammicus complex (Sauria: Iguanidae) in central Mexico: population cytogenetics. Syst Zool, 1986. 35 (3): p. 334-358.

11. Sites, J.W., Jr., N.H. Barton, and K.M. Reed, The genetic structure of a hybrid zone between two chromosome races of the Sceloporus grammicus complex (Sauria, Phrynosomatidae) in Central Mexico. Mol Ecol, 1995. 5: p. 379-392.

12. Barton, N.H. and G.M. Hewitt, Adaptation, speciation and hybrid zones. Nature, 1989. 341(6242): p. 497503.

13. Reed, K.M. and J.W. Sites, Jr., Female fecundity in a hybrid zone between two chromosome races of the Sceloporus grammicus complex (Sauria, Phrynosomatidae). Evolution, 1995. 49(1): p. 61-69.

14. Reed, K.M., J.W. Sites, Jr., and I.F. Greenbaum, Synapsis, recombination, and meiotic segregation in the mesquite lizard, Sceloporus grammicus, complex. II. Fission heteromorphism of the FM2 cytotype and evolution of chromosome 2. Cytogenet Cell Genet, 1992. 61(1): p. 46-54.

15. Sites, J.W., Jr. and I.F. Greenbaum, Chromosome Evolution in the Iguanid Lizard Sceloporus grammicus .II. Allozyme Variation. Evolution, 1983. 37(1): p. 54-65.

16. Dosselman, D.J., G.B. Schaalje, and J.W. Sites, Jr., An analysis of fluctuating asymmetry in a hybrid zone between two chromosome races of the Sceloporus grammicus complex (Squamata: Phrynosomatidae) in central Mexico. Herpetologica, 1998. 54(4): p. 434-447.

17. Tautz, D., Hypervariability of simple sequences as a general source for polymorphic DNA markers. Nucleic Acids Res, 1989. 17(16): p. 6463-6471.

18. Goldstein, D.B. and C. Schlötterer, Microsatellites: Evolution and Applications. 1999, New York: Oxford. p. v-vi.
19. Hancock, J.N., Microsatellites and other simple sequences: genomic context and mutational mechanisms, in Microsatellites: Evolution and Applications, D.B. Goldstein and C. Schlötterer, Editors. 1999, Oxford: New York. p. 1-9.

20. Chambers, G.K. and E.S. MacAvoy, Microsatellites: consensus and controversy. Comp Biochem Physiol B Biochem Mol Biol, 2000. 126(4): p. 455476.

21. Strassmann, J.E., et al., Strategies for finding and using highly polymorphic DNA microsatellite loci for studies of genetic relatedness and pedigrees, in Molecular Zoology: Advances, strategies and protocols, J. Ferraris and S. Palumbi, Editors. 1996, Wiley. p. 163180, 528-449.

22. Strand, M., et al., Destabilization of tracts of simple repetitive DNA in yeast by mutations affecting DNA mismatch repair. Nature, 1993. 365(6443): p. 274276.

23. Tautz, D. and C. Schlotterer, Simple sequences. Curr Opin Genet Dev, 1994. 4(6): p. 832-837.

24. Eisen, J.A., Mechanistic basis for microsatellite instability, in Microsatellites: Evolution and Applications, D.B. Goldstein and C. Schlötterer, Editors. 1999, Oxford: New York. p. 34-48.

25. Djian, P., Evolution of simple repeats in DNA and their relation to human disease. Cell, 1998. 94(2): p. 155-160.

26. Ionov, Y., et al., Ubiquitous somatic mutations in simple repeated sequences reveal a new mechanism for colonic carcinogenesis. Nature, 1993. 363(6429): p. 558-561.

27. Hamada, H., M.G. Petrino, and T. Kakunaga, A novel repeated element with Z-DNA-forming potential is widely found in evolutionarily diverse eukaryotic genomes. Proc Natl Acad Sci U S A, 1982. 79(21): p. 6465-9.

28. Hughes, C.R. and D.C. Queller, Detection of highly polymorphic microsatellite loci in a species with little allozyme polymorphism. Mol Ecol, 1993. 2(3): p. 131-137.

29. Wuthisuthimethaveea, S., et al., Development of microsatellite markers in black tiger shrimp (Penaeus monodon 
Fabricius). Aquaculture, 2003. 224: p. 39-50.

30. Oliveira, R.P., et al., Probing the genetic population structure of Trypanosoma cruzi with polymorphic microsatellites. Proc Natl Acad Sci U S A, 1998. 95(7): p. 3776-3780.

31. Longmire, J.L., D.C. Hahn, and J.L. Roach, Low abundance of microsatellite repeats in the genome of the brownheaded cowbird (Molothrus ater). J Hered, 1999. 90(5): p. 574-578.

32. Lagercrantz, U., H. Ellegren, and L. Andersson, The abundance of various polymorphic microsatellite motifs differs between plants and vertebrates. Nucleic Acids Res, 1993. 21(5): p. 1111-1115.

33. Gregory, T.R., Animal Genome Size Database. http://www.genomesize.com. 2001.

34. Olmo, E., Evolution of genome size and DNA base composition in reptiles. Genetica, 1981. 57: p. 39-50.

35. Sites, J.W., Jr., et al., A review of phylogenetic hypothesis for lizards of the genus Sceloporus (Phrynosomatidae): implications for ecological and evolutionary studies. Bull Am Mus Nat Hist, 1992. 213: p. 1-110.

36. Flores-Villa, O., et al., Multiple data sets, congruence, and hypothesis testing for the phylogeny of basal groups of the lizard genus Sceloporus (Squamata, Phrynosomatidae). Syst Biol, 2000. 49(4): p. 713-739.

37. Beckman, J.S. and J.L. Weber, Survey of human and rat microsatellites. Genomics, 1992. 12(4): p. 627-631.

38. Primmer, C.R., et al., Low frequency of microsatellites in the avian genome. Genome Res, 1997. 7(5): p. 471-482.

39. Moore, S.S., et al., The development and application of genetic markers for the Kuruma prawn Penaeus japonicus. Aquaculture, 1999. 173: p. 19-32.

40. Beaumont, M.A. and M.W. Bruford, Microsatellites in conservation genetics, in Microsatellites: Evolution and Applications, D.B. Goldstein and C. Schlötterer, Editors. 1999, Oxford: New York. p. 165-182.

41. Queller, D.C. and K.F. Goodnight, Estimation of genetic relatedness using allozyme data. Evolution, 1989. 43: p. 258-275.

42. Ostellari, L., et al., Novel microsatellite loci isolated from the northern krill, Meganyctiphanes norvegica (Crustacea, Euphausiacea). Mol Ecol, 2000. 9(3): p. 377-378.

43. Peters, J.M., Microsatellite loci for Pseudomyrmex pallidus (Hymenoptera:formicidae). Mol Ecol, 1997. 6(9): p. 887-888.

44. Chapuisat, M., Characterization of microsatellite loci in Formica lugubris $B$ and their variability in other ant species. Mol Ecol, 1996. 5(4): p. 599-601.

45. Lebas, N.R. and P.B. Spencer, Polymorphic microsatellite markers in the ornate dragon lizard, Ctenophorus ornatus. Mol Ecol, 2000. 9(3): p. 365366.

46. FitzSimmons, N.N., C. Moritz, and S.S. Moore, Conservation and dynamics of microsatellite loci over 300 million years of marine turtle evolution. Mol Biol Evol, 1995. 12(3): p. 432-440.

47. Johansson, M., H. Ellegren, and L. Andersson, Cloning and characterization of highly polymorphic porcine microsatellites. J. Hered, 1992. 83(3): p. 196-8.

48. Dib, C., et al., A comprehensive genetic map of the human genome based on 5,264 microsatellites. Nature, 1996. 380 (6570): p. 152-154.

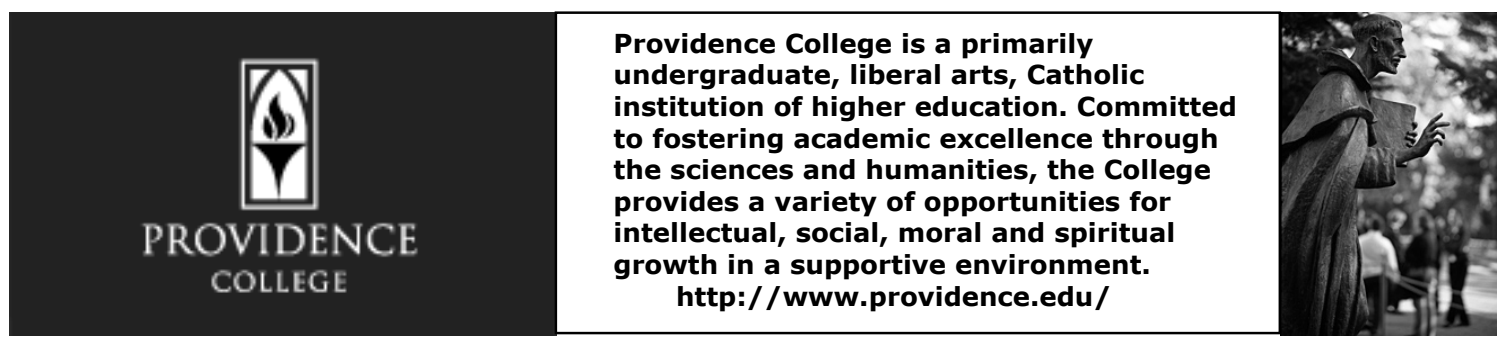


